\title{
Advantage of recombinant von Willebrand factor for peri-operative management in paediatric acquired von Willebrand syndrome
}

Dear Editors,

A 2-year-old African American male child with pulmonary valve stenosis sustained a skull fracture and large epidural haemorrhage after a fall. He underwent multiple procedures over several days, all of which were complicated by postoperative bleeding. Past bleeding history was significant for bleeding with eruption of new teeth. Family history was negative for bleeding manifestations. Laboratory workup prior to a planned cranioplasty revealed normal prothrombin time, activated partial thromboplastin time, thrombin time, fibrinogen, factor IX, factor XI and factor XIII (present by clot dissolution assay). Blood type was $\mathrm{AB}+$. A von Willebrand factor (vWF) panel revealed elevated factor VIII (FVIII) of 333\%, normal ristocetin cofactor activity (vWF:RCo) of 58\% (normal range 50\%-150\%) with vWF antigen (vWF:Ag) of $178 \%$ (normal range 50\%-150\%; Table 1). The vWF:RCo/ VWF:Ag ratio was abnormally decreased to 0.33 , consistent with acquired von Willebrand syndrome (avWS), likely secondary to pulmonic stenosis. Plasma von Willebrand multimer analysis revealed the absence or decreased abundance of the highest molecular weight multimers (HMWMs), but no definitely increased abundance of lower molecular weight vWF multimers, more characteristic of avWS than a congenital vWF defect. Although his VWF:RCo level was within a lownormal range, most guidelines suggest attaining a goal activity level of $100 \mathrm{IU} / \mathrm{dL}^{1}$ for surgical prophylaxis, particularly in major surgery.

Given the need for cranioplasty in the setting of avWS and significantly elevated factor VIII levels, there was concern about the use of traditional plasma-derived vWF concentrates (which all include FVIII in variable proportions) and potential for increased thrombotic risk. Although thrombotic events are rare occurrences in patients with bleeding disorders, the risk in von Willebrand disease (VWD) does seem to be increased compared to that seen in haemophilia. ${ }^{2}$ Three of the four vWD patients with thrombotic events within a systematic review whose FVIII level was measured had levels in excess of $200 \%{ }^{2}$ Given this, most guidelines recommend the following FVIII levels with replacement therapy and avoiding levels $>100 \%-200 \%{ }^{1,2}$ Thus, the decision was made to treat preoperatively with a recombinant vWF (rvWF) which does not contain FVIII (Vonvendi ${ }^{\circledR}$; Shire, Dublin, Ireland) and may confer a lower risk of thromboembolic sequelae. The rvWF $(80 \mathrm{lU} / \mathrm{kg}$ ) was administered just prior to surgery. Adequate haemostasis was achieved with minimal blood loss. There was concern for an acute bleed due to a drop in haemoglobin on postoperative day 2 , so he received an additional dose of $80 \mathrm{IU} / \mathrm{kg}$ of rvWF. This was later attributed to dilutional effects of intravenous fluid, and no evidence of bleeding was found by clinical assessment or imaging. The patient recovered well without complication.
Acquired von Willebrand syndrome is a rare entity with bleeding symptomatology similar to those seen in the inherited form of vWD. It is typically characterized by a negative family history, lack of prior bleeding symptoms and older age. It most commonly occurs in patients with lymphoproliferative or myeloproliferative disease (63\%), cardiovascular disease $(21 \%)$, solid tumours $(5 \%)$ or autoimmune disorders $(2 \%)$. Within cardiovascular disease, where loss of HMWM is caused by increased shear stress, it has been reported to occur with aortic stenosis, pulmonary stenosis, patent ductus arteriosus, ventricular and atrial septal defects and ventricular assist devices. ${ }^{3}$ The Risk of bleeding in patients with cardiopulmonary disorders has been associated with a VWF:RCo/VWF:Ag ratio of $<0.7 .^{4}$ There are no consistent guidelines for the treatment of aVWS likely due to its rarity. The efficacy of DDAVP is likely to be limited, and patients with cardiovascular disease have been reported to have the worst outcomes with its use with therapeutic responses only seen in $10 \%{ }^{5}$ Additionally, DDAVP provided additional obstacles in our patient given the haemostatic challenges inherent to neurosurgery, his young age and risk of hyponatremia, and concern for further elevation of his FVIII.

Another complicating factor in our patient was his baseline elevation in FVIII, likely secondary to a combination of an acute phase reaction to ongoing inflammation, non-O blood type ${ }^{6}$ and ethnicity. ${ }^{7}$ Elevated plasma levels of FVIII have been associated with a dosedependent increased thrombotic risk at levels $>150 \mathrm{IU} / \mathrm{dL} .{ }^{8}$ At least one case has been reported of avWS with elevated FVIII (to levels similar to our patient) treated with plasma-derived FVIII/vWF concentrate who went on to develop an embolic stroke. ${ }^{9}$ Given his severely elevated levels preoperatively, the use of a plasma-derived FVIII/ vWF concentrate was deemed to pose an unacceptable risk in terms of thrombosis. Recombinant vWF, in addition to the advantage of the absence of FVIII, also includes ultralarge and high-molecular weight multimers which are likely to be beneficial in the treatment of avWS. Additionally, most plasma-derived FVIII/VWF concentrates typically have a VWF:RCo/vWF:Ag ratio of $<1$, as opposed to $>1$ in rVWF. ${ }^{10}$ This is likely to be advantageous in a disease where the VWF:RCo/vWF:Ag ratio is by very definition decreased. This case illustrates an ideal scenario in which rvWF may provide distinct advantages over plasmaderived FVIII/vWF concentrates. This is, to our knowledge, the first report of the use of rVWF in a paediatric patient, as well as the first reported case in avWS, since its approval in 2015. As yet, it has only been approved for on-demand treatment, with prophylactic Phase 3 trials ongoing. Peri-operative treatment in this paediatric patient was efficacious, safe and tolerable. He did not develop any thrombotic complications, and no evidence of antibodies to vWF was observed. 


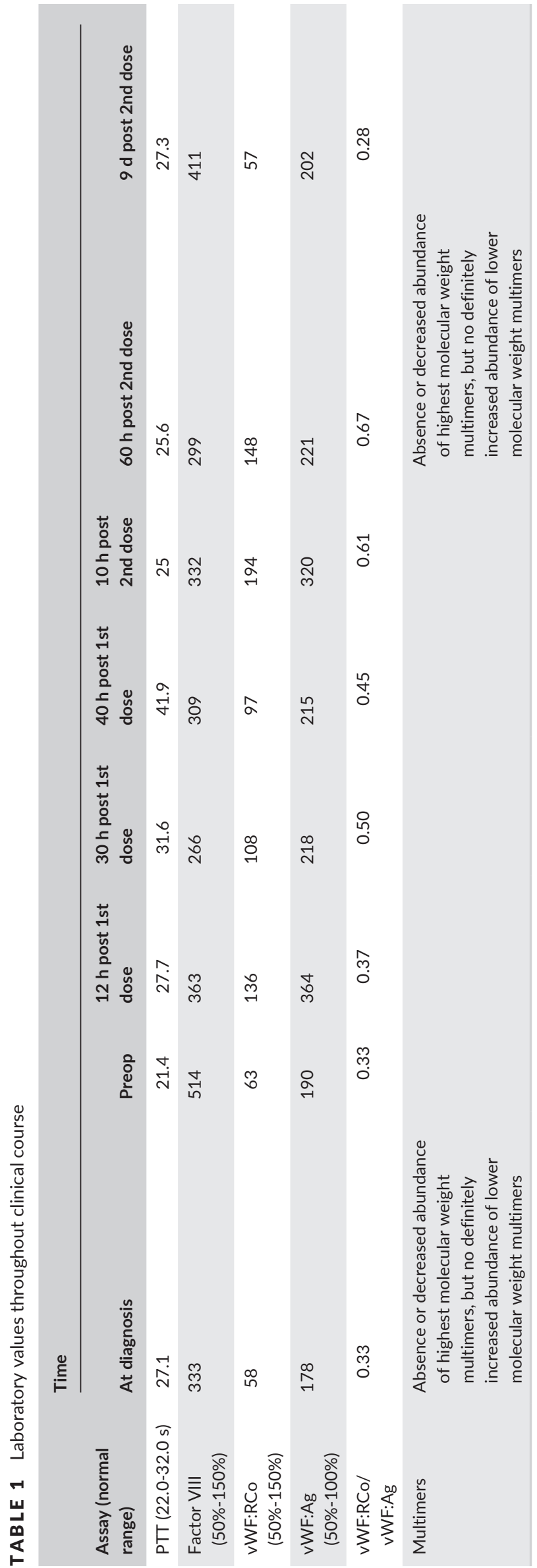

\section{DISCLOSURES}

ACW has served as a consultant for Shire and serves as a coinvestigator on a study funded by Shire. RJ has no conflicts to disclose. SWP has served as a consultant for Shire and CSL Behring.

\section{AUTHOR CONTRIBUTIONS}

ACW analysed the data and wrote the paper, RJ and SWP analysed the data and performed critical manuscript review and edits.

\section{ORCID}

A. C. Weyand (iD http://orcid.org/0000-0003-2595-8541

$$
\begin{array}{r}
\text { A. C. Weyand }{ }^{1} \text { iD } \\
\text { R. Jesudas }{ }^{2} \\
\text { S. W. Pipe }{ }^{1,3}
\end{array}
$$

${ }^{1}$ Department of paediatrics and Communicable Diseases, University of Michigan, Ann Arbor, MI, USA

${ }^{2}$ Bleeding and Clotting Disorders Institute, Peoria, IL, USA

${ }^{3}$ Department of Pathology, University of Michigan, Ann Arbor, MI, USA

Correspondence

Angela C. Weyand, Department of paediatrics and Communicable Diseases, University of Michigan, Ann Arbor, MI, USA.

Email: acweyand@med.umich.edu

\section{REFERENCES}

1. Nichols WL, Hultin MB, James AH, et al. von Willebrand disease (VWD): evidence-based diagnosis and management guidelines, the National Heart, Lung, and Blood Institute (NHLBI) Expert Panel report (USA). Haemophilia. 2008;14:171-232.

2. Coppola A, Franchini M, Makris M, Santagostino E, Di Minno G, Mannucci PM. Thrombotic adverse events to coagulation factor concentrates for treatment of patients with haemophilia and von Willebrand disease: a systematic review of prospective studies. Haemophilia. 2012;18:e173-e187.

3. Callaghan MU, Wong TE, Federici AB. Treatment of acquired von Willebrand syndrome in childhood. Blood. 2013;122:2019-2022.

4. Hashmi SK, Velasquez MP, Yee DL, Hui SK, Mahoney D Jr, Srivaths LV. paediatric acquired von Willebrand Syndrome in cardiopulmonary disorders: do laboratory abnormalities predict bleeding risk? J Pediatr Hematol Oncol. 2017;39:121-125.

5. Mital A. Acquired von Willebrand syndrome. Adv Clin Exp Med. 2016;25:1337-1344.

6. Yap ES, Timp JF, Flinterman LE, et al. Elevated levels of factor VIII and subsequent risk of all-cause mortality: results from the MEGA follow-up study. J Thromb Haemost. 2015;13:1833-1842.

7. Conlan MG, Folsom AR, Finch A, et al. Associations of factor VIII and von Willebrand factor with age, race, sex, and risk factors for atherosclerosis. The Atherosclerosis Risk in Communities (ARIC) Study. Thromb Haemost. 1993;70:380-385.

8. Jenkins PV, Rawley O, Smith OP, O'Donnell JS. Elevated factor VIII levels and risk of venous thrombosis. Br J Haematol. 2012;157:653-663.

9. Costello JP, Diab YA, Philippe-Auguste M, et al. Acquired von Willebrand syndrome in a child following Berlin Heart EXCOR paediatric Ventricular Assist Device implantation: case report and concise literature review. World J Pediatr Congenit Heart Surg. 2014;5:592-598.

10. Franchini M, Mannucci PM. Von Willebrand factor (Vonvendi(R)): the first recombinant product licensed for the treatment of von Willebrand disease. Expert Rev Hematol. 2016;9:825-830. 